\title{
Operation and output pulse characteristics of an extremely compact capillary-discharge tabletop soft-x-ray laser
}

\author{
B. R. Benware, C. H. Moreno, D. J. Burd, and J. J. Rocca \\ Department of Electrical Engineering, Colorado State University, Fort Collins, Colorado 80523
}

Received January 2, 1997

\begin{abstract}
We report on the operating parameters and laser output pulse characteristics of an extremely compact discharge-pumped 46.9-nm laser of a size comparable with that of many widely utilized visible and ultraviolet gas lasers. This capillary-discharge laser generated subnanosecond laser pulses with energies of as much as $25 \mu \mathrm{J}$ by single-pass amplification in an 18.6-cm-long argon-plasma column. Measurements of the laser output energy, pulse width, far-field beam profile, and beam divergence are reported. ( 1997 Optical Society of America
\end{abstract}

There is significant interest in the development of compact soft-x-ray lasers for applications. ${ }^{1-3}$ The development of tabletop soft-x-ray laser sources that are simple to operate is expected to have an important impact in several disciplines, including surface science, photophysics and photochemistry, and biology. Several ultrashort-wavelength laser excitation schemes that utilize as pump sources compact lasers with pulse widths ranging from femtoseconds to nanoseconds are currently being investigated. ${ }^{4-8}$ In particular, significant progress has been made in the past two years with ultrashort laser pulses. ${ }^{4-8}$

Our approach consists of generating the lasing media by direct discharge excitation. ${ }^{9-11}$ In this excitation scheme elongated plasma columns are generated in capillary channels by fast discharge excitation. The fast current pulse rapidly compresses the capillary plasma to form a dense and hot column with a length-to-diameter ratio approaching 1000:1. During the final stage of the compression, the necessary plasma conditions for soft-x-ray amplification by collisional excitation of multiply ionized atoms are obtained. The implementation of this scheme resulted in the first demonstration of large soft-x-ray amplification in a discharge-created plasma ${ }^{10}$ in the 46.9-nm line of Ne-like Ar. Subsequent optimization of the Ne-like Ar laser resulted in gains of as much as $\exp (27)$ and in saturated operation of the amplifier. ${ }^{11}$ Recently we also obtained an amplification of $\exp (7.5)$ in Ne-like S at $60.8 \mathrm{~nm} .{ }^{12}$ In those proof-of-principle experiments, no effort was made to optimize the laser output or minimize the size of the device.

In this Letter we report on the laser output pulse characteristics and the operating parameters of an extremely compact $46.9-\mathrm{nm}$ Ne-like Ar capillarydischarge soft-x-ray laser. This laser, which is comparable in size with many widely utilized visible and ultraviolet gas lasers, produced laser pulses with energies of as much as $25 \mu \mathrm{J}$ by single-pass amplification through the capillary channel. This energy is approximately four times larger than what we previously reported at this wavelength for single-pass amplification. The results demonstrate the feasibility of developing extremely compact and practical discharge-pumped ultrashort-wavelength lasers for applications.

The laser is shown in Fig. 1 in comparison with a $5-\mathrm{mW} \mathrm{He}-\mathrm{Ne}$ laser. Lasing is produced when a 4-mm-diameter polyacetal capillary channel filled with preionized pure Ar gas is excited with the current pulse shown in Fig. 2. The current pulse, which has an amplitude of $\approx 37 \mathrm{kA}$ and a first half-cycle duration of $\sim 70 \mathrm{~ns}$, is created by discharge of a water dielectric Blumlein transmission line through a spark gap pressurized with $\mathrm{SF}_{6}$. The transmission line is pulse charged by a four-state Marx generator. The Marx generator is contained in a separate box that sits on the floor and is connected to the laser head shown in Fig. 1 with a coaxial cable. We maintained an Ar pressure of $\sim 600$ mTorr in the capillary by continuously injecting Ar and differentially pumping it with a turbomolecular pump.

The temporal evolution and the energy of the laser pulse were measured with a vacuum photodiode with an aluminum photocathode, which was positioned on axis with the capillary at a distance of $150 \mathrm{~cm}$ from the exit of the plasma column. Figure 2 shows the temporal evolution of the axially emitted radiation in relation to the current pulse for an 18.6-cm-long capillary. The onset of the laser pulse occurs at $\sim 39 \mathrm{~ns}$ after the beginning of the current pulse with shot-to-shot fluctuations of

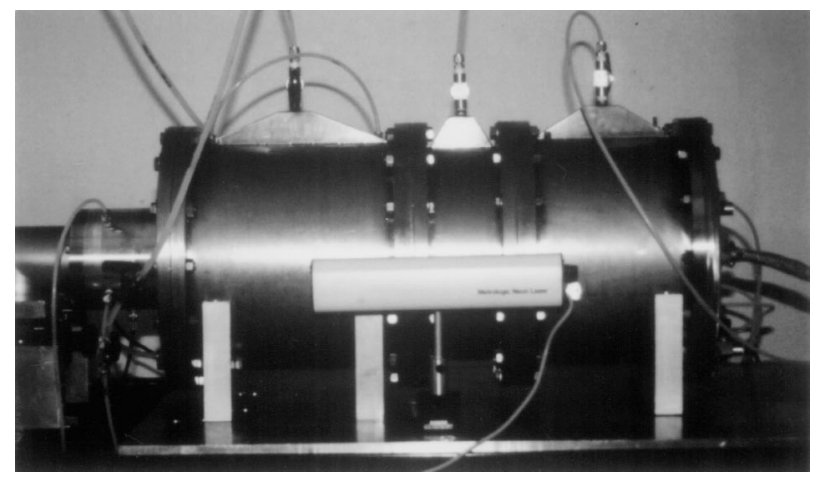

Fig. 1. Size of the 46.9-nm laser (back) relative to a $5-\mathrm{mW}$ $\mathrm{He}-\mathrm{Ne}$ laser (front). 


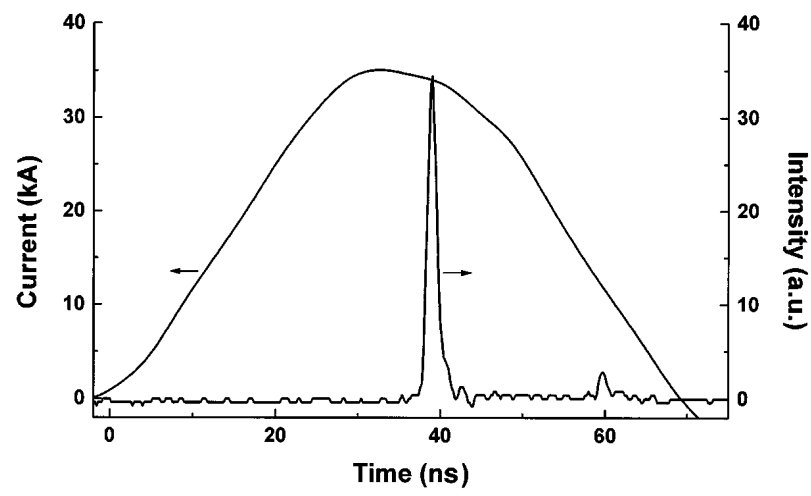

Fig. 2. Discharge current pulse used to excite an 18.6-cmlong capillary and a corresponding 46.9-nm laser output pulse. The current pulse was measured with a Rogowsky coil. The intensity peak at $60 \mathrm{~ns}$ is a reflection of the electrical signal produced by the laser pulse in the photodiode.

less than $\pm 0.2 \mathrm{~ns}$. It should be noticed that under optimized conditions the intensity of the spectrally integrated soft-x-ray incoherent radiation emitted in the solid angle of the detector is negligible in comparison with that of the laser pulse. Figure 3 shows the temporal profile of a typical laser pulse. The laser pulse widths were measured to range from 0.6 to $0.7 \mathrm{~ns}$.

The laser pulse energy that was generated was measured for capillaries of lengths ranging from 8.7 to $18.6 \mathrm{~cm}$. To avoid saturation of the photodiode, we attenuated the laser beam with stainless-steel meshes of calibrated transmissivity. Since the objective of these measurements was to determine the laser energy that can be obtained with this device rather than to obtain an accurate measurement of the gain, the current pulse width was not kept strictly constant for all capillary lengths. As illustrated in Fig. 4, the laser output energy increased from $\sim 0.3 \mu \mathrm{J}$ for an 8.7-cm-long plasma to as much as $25 \mu \mathrm{J}$ for 18.6 -cm-long plasma columns. In the longest capillaries the laser intensity reaches the saturation intensity level several centimeters before the end of the plasma column, allowing for efficient energy extraction. The measured energy values correspond to the laser photon fluxes that reach the photodiode. Since the beam is partially attenuated by neutral-gas absorption, the energy can be expected to be higher at the exit of the amplifier. Double-pass amplification would produce laser pulses with higher energy ${ }^{11}$ but mirrors deteriorate rapidly when placed in close proximity to the plasma. Therefore, the singlepass generation of laser pulses with substantial energy and power that is reported here (as much as $25 \mu \mathrm{J}$, $\approx 40 \mathrm{~kW}$ ) is of practical interest for repetitive operation.

The laser was repeatedly fired at a rate of up to 2 shots/min, for as many as 300 shots in a single capillary. Figure 5 shows the shot-to-shot laser output intensity fluctuations from a 12.4-cm-long capillary fired at 30 -s intervals. The fluctuations in the intensity are more a result of variations in the formation of the plasma column than a consequence of shot-toshot variations in the peak magnitude of the current pulse. The laser output power slowly decreases as a function of the number of shots in a new capillary, as the capillary deteriorates with use owing to discharge ablation of the walls. High output power was typically obtained for the first 50 to 100 shots in a new capillary. Nevertheless, lasing at degraded output energies was observed for at least 300 shots.

We obtained a direct measurement of the beam divergence by recording the far-field pattern of the laser beam $160 \mathrm{~cm}$ from the exit of the capillary. The laser

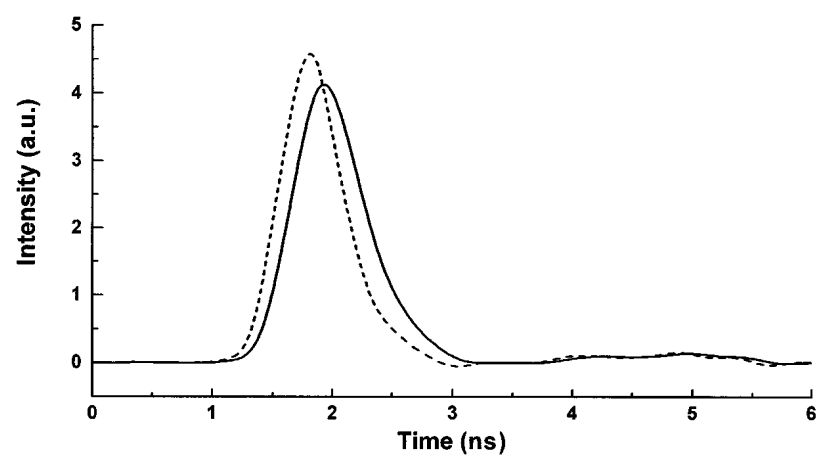

Fig. 3. Temporal evolution of the laser intensity. The solid curve is the photodiode signal recorded with a 1-GHz-bandwidth analog oscilloscope. The dashed curve shows the signal corrected for the limited bandwidth of the detection system.

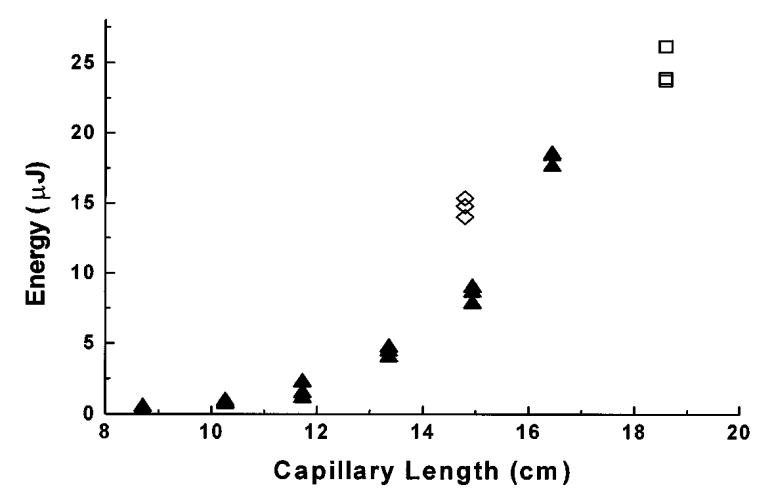

Fig. 4. Laser output pulse energy produced with capillaries of various lengths. The best three shots obtained for each capillary length are shown. Average values are lower. For typical shot-to-shot energy fluctuations, see Fig. 5. Different symbols represent shots in different capillaries.

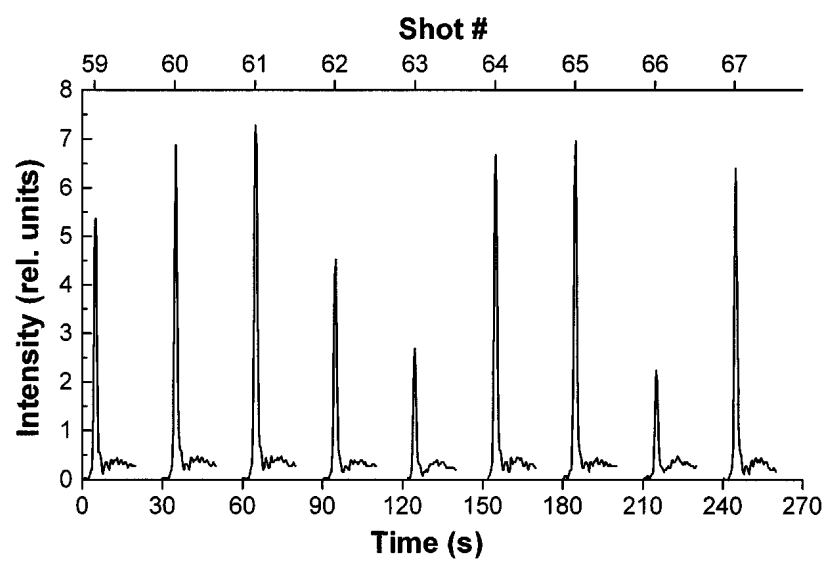

Fig. 5. Laser output obtained from a 12.4-cm-long capillary, firing the discharge at 30 -s intervals. The shot number identifies the number of shots in the same capillary. 


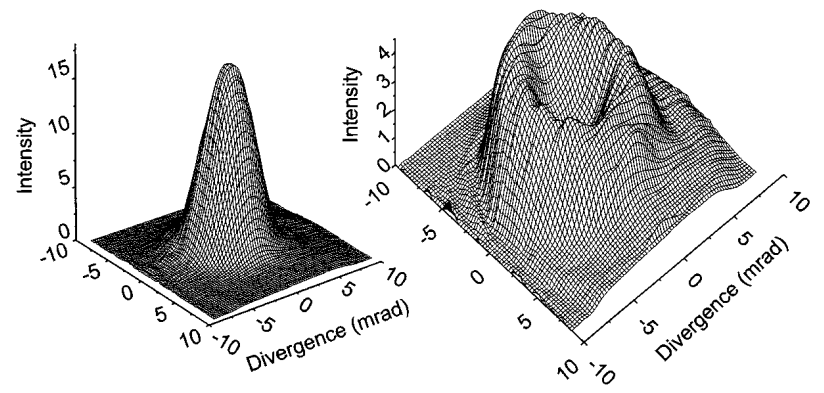

Fig. 6. Far-field laser beam profiles.

beam profiles were recorded by a multichannel-plate intensified CCD detector. The multichannel plate was gated to record the laser beam profile more clearly by avoiding the background caused by the long-lasting spontaneous emission radiated by the plasma column. Figure 6 illustrates the results of the beam-profile measurements for a capillary $14.8 \mathrm{~cm}$ in length. Two different characteristic beam profiles were observed, one a smooth profile with maximum intensity on axis and a second with an annular intensity distribution. The first was measured to have a beam divergence of $3.5 \pm 1 \mathrm{mrad}$. The doughnut-shape profile, which has a larger divergence, could correspond to plasma columns that have larger density gradients. The correspondingly larger variation of the index of refraction deflects the rays away from the axis of the plasma column, causing the observed ring structure in the far field. ${ }^{13,14}$ The angular separation between the radially opposite peaks of the annular profile is typically $6.5 \pm 1 \mathrm{mrad}$.

In summary, we have obtained 46.9-nm laser pulses with energies of as much as $25 \mu \mathrm{J}$ and powers of as much as $40 \mathrm{~kW}$ with an extremely compact dischargepumped tabletop amplifier. The laser pulse width, far-field beam profile, and beam divergence have also been measured. This is to our knowledge the first demonstration of a soft-x-ray laser of a size comparable with that of many widely utilized optical and ultraviolet lasers that is capable of producing substantial output pulse energy. These results are expected to lead to the use of very compact and practical discharge-pumped ultrashort wavelength lasers in applications.

We thank J. L. A. Chilla, F. G. Tomasel, M. C. Marconi, and V. N. Shlyaptsev for helpful assistance. This research was supported by the National Science Foundation. We also acknowledge the support of the Colorado Advance Technology Institute for a collaboration with Hyperfine, Boulder, Colo.

\section{References}

1. S. Svanverg and C. G. Wahlstrom, eds., Proceedings of the Fifth International Conference on X-Ray Lasers (Institute of Physics, Bristol, UK, 1995).

2. J. J. Rocca and P. L. Hagelstein, eds., Soft X-Ray Lasers and Applications, Proc. SPIE 2520 (1995).

3. D. Eden and D. Matthews, eds., X-Ray Lasers 1994, Fourth International Colloquium AIP Conf. Proc. 332 (1994).

4. B. Lemoff, G. Y. Yin, C. L. Gordon III, C. P. J. Barty, and S. E. Harris, Phys. Rev. Lett. 74, 1574 (1995).

5. P. V. Nickles, M. Schmurer, M. P. Kalashnikov, I. Will, W. Sanders, and V. N. Shlyaptsev, Proc. SPIE 2520, 373 (1995).

6. Y. Nagata, K. Midorikawa, S. Kubodera, M. Obara, H. Tashiro, and K. Toyoda, Phys. Rev. Lett. 71, 3774 (1993).

7. D. Korobkin, C. Nam, and S. Suckewer, Phys. Rev. Lett. 77, 5206 (1996).

8. J. G. Goodberlet, S. Basu, and P. L. Hagelstein, J. Opt. Soc. Am. B 12, 980 (1995).

9. J. J. Rocca, O. D. Cortazar, B. Szapiro, K. Floyd, and F. G. Tomasel, Phys. Rev. E 47, 1299 (1993).

10. J. J. Rocca, V. Shyaptsev, F. G. Tomasel, O. D. Cortazar, D. Hartshorn, and J. L. A. Chilla, Phys. Rev. Lett. 73, 2192 (1994).

11. J. J. Rocca, D. P. Clark, J. L. A. Chilla, and V. N. Shlyaptsev, Phys. Rev. Lett. 77, 1476 (1996).

12. F. G. Tomasel, J. J. Rocca, V. N. Shlyaptsev, and C. D. Macchietto, Phys. Rev. A 55, 1437 (1997).

13. R. A. London, Phys. Fluids 31, 184 (1988).

14. J. L. A. Chilla and J. J. Rocca, J. Opt. Soc. Am. B 13, 2841 (1996). 